\title{
Plasmon resonances of silver nanowires with a nonregular cross section
}

\author{
Jörg P. Kottmann and Olivier J. F. Martin* \\ Electromagnetic Fields and Microwave Electronics Laboratory, Swiss Federal Institute of Technology, \\ ETH-Zentrum, 8092 Zurich, Switzerland \\ David R. Smith and Sheldon Schultz \\ Department of Physics, University of California, San Diego, 9500 Gilman Drive, La Jolla, California 92093-0319
}

(Received 30 April 2001; published 8 November 2001)

\begin{abstract}
We investigate numerically the spectrum of plasmon resonances for metallic nanowires with a nonregular cross section, in the 20-50 $\mathrm{nm}$ range. We first consider the resonance spectra corresponding to nanowires whose cross sections form different simplexes. The number of resonances strongly increases when the section symmetry decreases: A cylindrical wire exhibits one resonance, whereas we observe more than five distinct resonances for a triangular particle. The spectral range covered by these different resonances becomes very large, giving to the particle-specific distinct colors. At the resonance, dramatic field enhancement is observed at the vicinity of nonregular particles, where the field amplitude can reach several hundred times that of the illumination field. This near-field enhancement corresponds to surface-enhanced Raman scattering (SERS) enhancement locally in excess of $10^{12}$. The distance dependence of this enhancement is investigated and we show that it depends on the plasmon resonance excited in the particle, i.e., on the illumination wavelength. The average Raman enhancement for molecules distributed on the entire particle surface is also computed and discussed in the context of experiments in which large numbers of molecules are used.
\end{abstract}

DOI: 10.1103/PhysRevB.64.235402

PACS number(s): 73.20.Mf, 42.25.Fx, 42.68.Mj, 78.30.-j

\section{INTRODUCTION}

Nanosized metal particles with subwavelength dimensions exhibit a wealth of optical phenomena directly related to geometry-dependent surface plasmon resonances that can be excited in response to applied electromagnetic fields. Plasmon resonances lead to large scattering cross sections (SCS's) at specific wavelengths in many metal nanoparticles, including those made of silver and gold, and are responsible for the characteristic color associated with suspensions of colloidal metal particles. The connection between the scattered spectrum of a nanoparticle and its physical properties was established long ago; medieval artisans, for example, made use of metal colloidal particles in the production of certain types of stained glass. ${ }^{1}$

In addition to their large SCS's, plasmon resonant particles have very large and very localized local electromagnetic fields. These local fields play a key role in surfaceenhanced Raman scattering (SERS), wherein the Raman spectrum of a molecule near a nanoparticle surface is enhanced by several orders of magnitude. ${ }^{2,3}$ This enhancement can be large enough that the Raman spectrum of a single molecule can be acquired. ${ }^{4-6}$ The enhanced local fields of plasmon resonant particles are useful for a variety of applications, including biosensors, ${ }^{7-9}$ as sources for nanolithography ${ }^{10}$ and as probes in scanning near-field optical microscopy (SNOM). ${ }^{11,12}$ Finally, their spectral selectivity may make plasmon resonant particles a key component in future passive optical devices based on evanescent optical transport $^{13-15}$ or even in newly proposed active optical components. $^{16}$

In recent years, considerable progress has been made in the fabrication of metallic nanostructures in a controlled manner, including features in the $10-50 \mathrm{~nm}$ range. ${ }^{17-26} \mathrm{Me}-$ tallic particles with a variety of shapes and dimensions are now readily available for experiments. A thorough understanding of the detailed local fields associated with plasmon resonant particles is therefore warranted, to enhance the design and optimization of applications based on these particles. However, an accurate solution of the local fields of plasmon resonant particles of arbitrary shape remains a theoretical challenge. Analytical solutions for the fields are known only for particles with a very simple shape, like that of a sphere or an ellipsoid, ${ }^{27-35}$ or spherical shells and periodic cylinder gratings. ${ }^{36-44}$ While electrostatic methods can provide some level of insight, ${ }^{45}$ complete electromagnetic solvers are needed to obtain accurate results. Many groups have developed methods of solving Maxwell's equations to investigate nonregular plasmon resonant particles in the 100-200 nm size range, ${ }^{46-48}$ however, although particles of this size provide large SCS's at the plasmon resonance, the resonances are very broad and the field enhancement in the vicinity of the particles is relatively small.

Recently we have presented a numerical approach for the solution of the fields associated with plasmon resonant nanoparticles of arbitrary two-dimensional geometry, which leads to highly accurate, converged solutions, even for particles having extremely large local enhancement and field variation. ${ }^{49}$ Recently we demonstrated that nanowires with a triangular section had a very complex spectrum of plasmon resonances. ${ }^{50}$ For sections smaller than $50 \mathrm{~nm}$, we observed many distinct, narrow resonances, associated with strong near-field distributions. In Ref. 51 we illustrated with movies the spectral response of triangular particles and demonstrated that a strong near-field enhancement was obtained for particles smaller than about $50 \mathrm{~nm}$. The topology of these different resonances was correlated to the polarization charges distributions induced in these particles in Ref. 52. 
The objective of the present publication is to study in detail the relation between the cross-sectional shape of a nanowire and its resonance spectrum. In Sec. II we recall our formalism and address some of the specific issues related to the accurate simulation of plasmon resonances. In Sec. III we investigate the resonance spectrum corresponding to nanowires whose cross sections form different simplexes and consider the implication of these results for SERS. We present our conclusions in Sec. IV.

\section{FORMALISM}

\section{A. Theory}

The electromagnetic scattering properties of metal nanoparticles can be described by solving Maxwell's equations. ${ }^{53,54}$ In this model, the complete description of the material properties of the metal is encompassed in the dispersion relation, which gives the complex permittivity $\varepsilon(\omega)$ as a function of the frequency (or wavelength). Near the metal plasma frequency $\omega_{\mathrm{p}}$ of the metal, the dispersion relation is governed by the interaction between light and the conduction electron gas or expressed with their quantum counterparts by the photon-plasmon interaction. The combined exciton is often referred to as a plasmon polariton. For certain metals such as silver, copper, and gold, $\omega_{\mathrm{p}}$ is in the visible frequency range. At specific negative permittivity values, plasmon resonances will be excited in these small particles. These specific permittivity values strongly depend on the particle size and shape, since the boundary conditions imposed by Maxwell's equations determine whether such a particle resonance can build up. These resonances are often referred to as the surface modes of the particle. ${ }^{55}$

The plasmon resonances are analytically known only for simple geometries, such as a sphere or a cylinder. ${ }^{53}$ In a very small sphere, for instance, one single resonance can be excited, when $\varepsilon=-2(\mathrm{Ag}: \lambda \simeq 355 \mathrm{~nm}, \mathrm{Au}: \lambda \simeq 490 \mathrm{~nm})$, whereas a cylinder is in resonance when $\varepsilon=-1$ (Ag: $\lambda$ $\simeq 337 \mathrm{~nm}, \mathrm{Au}: \lambda \simeq 253 \mathrm{~nm}$ ). With increasing particle size, these resonances are redshifted and broadened, and additional higher-order resonances can be excited. ${ }^{53}$

More than one single resonance can be excited in a nonregular structure, irrespective of its size. A small ellipse, for instance, exhibits two resonances, corresponding to the illumination directions along and normal to its major axis. Recently we demonstrated that triangular nanoparticles have several resonances for each illumination direction. ${ }^{50}$

Whether these different modes of a nanoparticle of arbitrary shape can be resolved strongly depends on the material absorption (the imaginary part of $\varepsilon$ ): large absorption broadens the resonances and can result in a nearly featureless band. Silver, compared with other metals that have their plasma frequency in the optical range (e.g., gold and copper), has a comparatively low absorption and thus narrower resonances.

Another phenomenon that can arise at wavelengths where the permittivity changes its sign is the excitation of longitudinal plasmons. ${ }^{54,56,57}$ However, this is a very weak effect, and for flat boundaries this mode is hardly excited. ${ }^{54}$
One may wonder whether the classical description of the material that we use, based solely on Maxwell's theory and a local dispersion relation, is appropriate for the small structures investigated here. Actually, it has been experimentally shown that this macroscopic approach is adequate for particle dimensions as small as $2 \mathrm{~nm} .{ }^{35,54,58-60}$ Quantum effects must only be taken into account for smaller particles, using for example a jellium or quantum-chemical model..$^{54,61-63}$ For particles in the $2-20 \mathrm{~nm}$ range, the dispersion relation depends noticeably on the particle geometry, since the electron mean free path decreases as electron scattering at the surface becomes more important. ${ }^{35,58-60}$ However, for the silver particles investigated here, only the imaginary part of the permittivity increases slightly, and the bulk values of permittivity still represent a good approximation. This point will be addressed in greater detail in Sec. III C.

\section{B. Model}

As established in the previous section, Maxwell's equations are well suited to study the plasmon resonances of silver particles in the $20-50 \mathrm{~nm}$ range, and we shall use the experimentally obtained permittivity values from Johnson and Christie. ${ }^{64}$ For nonmagnetic media, Maxwell's equations reduce in the frequency domain to the vectorial wave equation, which is formally solved by the volume integral equation

$$
\begin{aligned}
\mathbf{E}(\mathbf{r} ; \omega)= & \mathbf{E}^{0}(\mathbf{r} ; \omega)+\int_{\mathrm{V}} \mathrm{d} \mathbf{r}^{\prime} \mathbf{G}^{\mathrm{B}}\left(\mathbf{r}, \mathbf{r}^{\prime} ; \omega\right) \\
& \cdot k_{0}^{2}\left[\varepsilon\left(\mathbf{r}^{\prime} ; \omega\right)-\varepsilon_{\mathrm{B}}\right] \mathbf{E}\left(\mathbf{r}^{\prime} ; \omega\right) .
\end{aligned}
$$

Here $\mathbf{E}_{0}(\mathbf{r} ; \omega)$ is the incident electric field with vacuum wave number $k_{0}, \mathbf{E}(\mathbf{r} ; \omega)$ is the unknown total scattered field, $\varepsilon(\mathbf{r} ; \omega)$ the particle permittivity, and $\varepsilon_{\mathrm{B}}$ that of the background. The dyadic $\mathbf{G}^{\mathrm{B}}\left(\mathbf{r}, \mathbf{r}^{\prime} ; \omega\right)$ is the Green's tensor associated with the infinite homogeneous background. ${ }^{65}$ Note that this formalism can also be used when the background is a surface or a stratified medium. ${ }^{66,67}$

We study two-dimensional (2D) silver particles, i.e., particles having a translation symmetry along the third (not shown) direction, such as nanowires or infinitely long nanorods. The particles are illuminated in the plane of the figures with the electric field in the same plane (transverse-electric polarization). For transverse-magnetic polarization, where the incident magnetic field is in the plane, plasmons cannot be excited.

We solve Eq. (1) with a newly developed technique based on finite elements. ${ }^{49}$ The arbitrary particle section is discretized using triangles, and the unknown field $\mathbf{E}(\mathbf{r} ; \omega)$ is expanded into a sum of basis functions defined on each triangle. A Galerkin test procedure is then used to obtain a system of algebraic equations for the unknown field. We refer the reader to Ref. 49 where this technique is described in detail.

Since the spectrum of resonances for nonregularly shaped particles is not known, it was very important to first assess the accuracy of this technique. In Refs. 49 and 50 we compared the results from our numerical approach with analyti- 


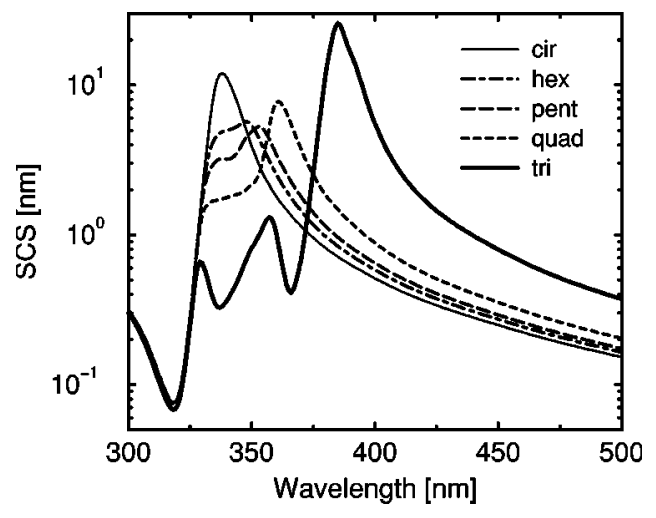

FIG. 1. SCS's for the $20 \mathrm{~nm}$ simplexes.

cal reference solutions for simple geometries. The agreement between the solutions was exact. A key component in achieving these high-accuracy results was a new regularization scheme that we developed to extract the singularity of the Green's tensor. This regularization scheme was further extended to neighboring elements, which considerably improves the accuracy. ${ }^{49}$

The particle cross sections are discretized with 20003000 triangular elements. We verified that this discretization number was large enough that the numerical results did not depend on it. To provide a more realistic model of a realizable nanoparticle, and to avoid numerical difficulties, all the particle corners are rounded off by $0.25 \mathrm{~nm}$. This point will be discussed in detail in Sec. III D.

\section{NUMERICAL RESULTS}

\section{A. Simplexes}

Knowing that a cylindrical silver particle exhibits only one resonance, while a complex triangular particle exhibits at least five resonances, ${ }^{50}$ we anticipate that the resonance spectrum should increase in complexity as the cross section of the particle is reduced from one of high symmetry to one of lower symmetry. In this section, we investigate the plasmon resonances of small silver wires with varying simplex cross section.

Figure 1 shows the scattering cross sections from nanowires having cross sections corresponding to that of a circle, a hexagon, a pentagon, a square, and a triangle. The diameter of the circle is $20 \mathrm{~nm}$, and all the particles have the same area, so that the SCS's should be comparable. The illumination direction is along one of the particle's symmetry axis.

For the circle we recover the well-known result of a single resonance, at $\lambda=338 \mathrm{~nm}$ (corresponding to $\varepsilon=-1.07$ $+0.29 i)$. The full width half maximum (FWHM) of the resonance is about $25 \mathrm{~nm}$. As can be seen in Fig. 1, the structure of the SCS's becomes more complex for less regular particles. The main resonance is redshifted from $\lambda$ $=338 \mathrm{~nm}$ (circle) to $\lambda=350 \mathrm{~nm}$ (hexagon), $\lambda=357 \mathrm{~nm}$ (pentagon), $\lambda=361 \mathrm{~nm}$ (square), and $\lambda=385 \mathrm{~nm}$ (triangle). An additional resonance appears for all the noncircular particles. Moreover, a third resonance can be observed for the

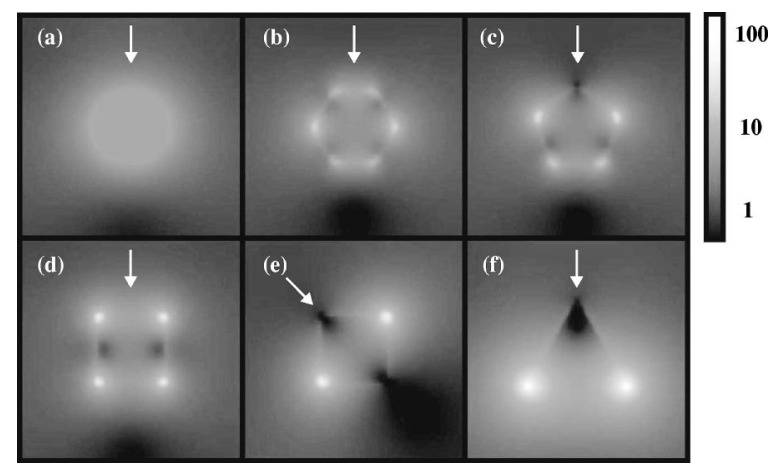

FIG. 2. Relative field amplitude distribution for the $20 \mathrm{~nm}$ simplexes, at their respective main resonance (see Fig. 1). The arrow indicates the propagation direction of the illumination field.

triangle at $\lambda=357 \mathrm{~nm}$. The influence of the direction of illumination on the SCS's is very small due to the symmetry of the simplexes (not shown).

The large SCS's that occur at the plasmon resonances are associated with large field amplitudes at the vicinity of the particle. In Fig. 2 we show this near-field amplitude distribution for the main resonance of each simplex. The amplitude is normalized to the incident amplitude and the illumination direction indicated by the arrow.

The amplitude inside the circular particle is almost homogeneous, about 7 times the incident field. The boundary of the cylinder is not visible in the amplitude distribution because the resonance happens when $\varepsilon$ is close to -1 . Maxwell's boundary conditions impose then that the field amplitude is continuous. ${ }^{68}$ The field amplitude decays rapidly outside the particle.

The field becomes strongly heterogeneous for the nonregular structures. At the main resonance, the field amplitude takes large values at the corners transverse to the incident wave vector (Fig. 2). There the relative field amplitude exceeds 20 for the hexagon and the pentagon, 70 for the square, and 150 for the triangle. Let us stress here that the large fields we observe near the corners are not produced by the lightning rod effect. The latter provides only a field amplification factor in the order of 5-10, even for very large permittivity values, as illustrated in Fig. 4(c) of Ref. 51.

In Fig. 3 we show the near-field distributions for the triangle corresponding to the three resonances observed in Fig. 1 . For the main resonance $(\lambda=385 \mathrm{~nm})$ and the resonance at $\lambda=358 \mathrm{~nm}$ we observe very large fields near the corners transverse to the illumination direction. However, the field distributions have a very different topology for each reso-

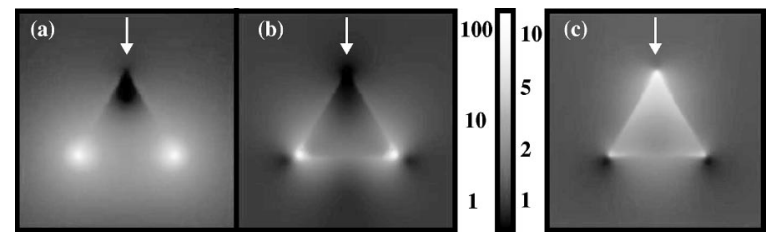

FIG. 3. Relative field amplitude distribution associated with the three resonances of the triangle (Fig. 1). (a) Main resonance, $\lambda$ $=385 \mathrm{~nm}$; (b) $\lambda=358 \mathrm{~nm}$; and (c) $\lambda=329 \mathrm{~nm}$. 


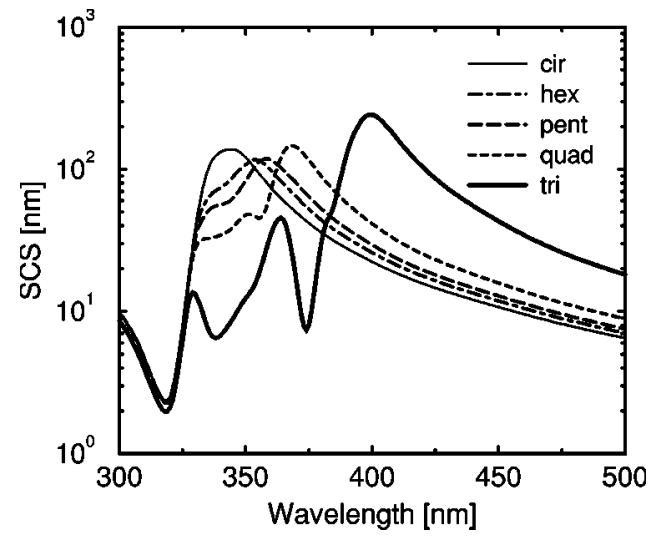

FIG. 4. SCS for the $50 \mathrm{~nm}$ simplexes.

nance, which can easily be understood in terms of polarization charges. ${ }^{50}$ These two resonances correspond to what is also known as the particle surface modes of order 0 and $1 .^{45,53}$ The polarization charge topology of the main resonance (order 0 ) is the most simple one, with charges of a given sign near each corner, leading to a pointlike field distribution at the corners, as observed in Fig. 3(a). On the other hand, charges of both signs accumulate near the corner for the resonance at $\lambda=385 \mathrm{~nm}$ (order 1), leading to a dipolelike field distribution around the corners, as observed in Fig. 3(b).

A different amplitude scale is used in Fig. 3(c) for the resonance at $\lambda=329 \mathrm{~nm}$. Although the maximum field amplitude is comparatively small (10 times the incident field), this resonance is most remarkable: The field is maximum at the corner longitudinal to the illumination direction. As discussed in Ref. 51, this resonance does not appear to be redshifted for larger particle sizes, contrary to the surface modes Figs. 3(a) and 3(b). This resonance is likely related to the bulk longitudinal mode, which appears in the bulk when the illumination frequency and the electron plasma frequency $\omega_{\mathrm{p}}$ coincide (i.e., when $\varepsilon \simeq 0$ ). ${ }^{55}$

In Fig. 4, we show the SCS diagram for larger simplexes. All the particles have again the same area, the diameter of the circle being now $50 \mathrm{~nm}$. We observe mainly two differences with respect to the SCS's of the smaller simplexes (Fig. 1): First, for all shapes the main resonance is redshifted (e.g., for the triangle it is now at $\lambda=399 \mathrm{~nm}$, compared to $\lambda=385 \mathrm{~nm}$ for the $20 \mathrm{~nm}$ triangle). Second, the resonance FWHM is roughly doubled (i.e., for the circle, it is now more than $50 \mathrm{~nm}$, compared to $25 \mathrm{~nm}$ previously). We also see in Fig. 3 that an additional resonance can be resolved for the square at $\lambda=351 \mathrm{~nm}$, whereas two additional resonances start to emerge for the triangle at approximately $\lambda$ $=350 \mathrm{~nm}$ and $\lambda=382 \mathrm{~nm}$.

\section{B. Implications for SERS}

In this section we will focus on the near-field distribution and discuss practical implications for SERS. The utilization of this electromagnetic enhancement for scanning near-field optical microscopy has been discussed in Ref. 69 and shall not be discussed here.

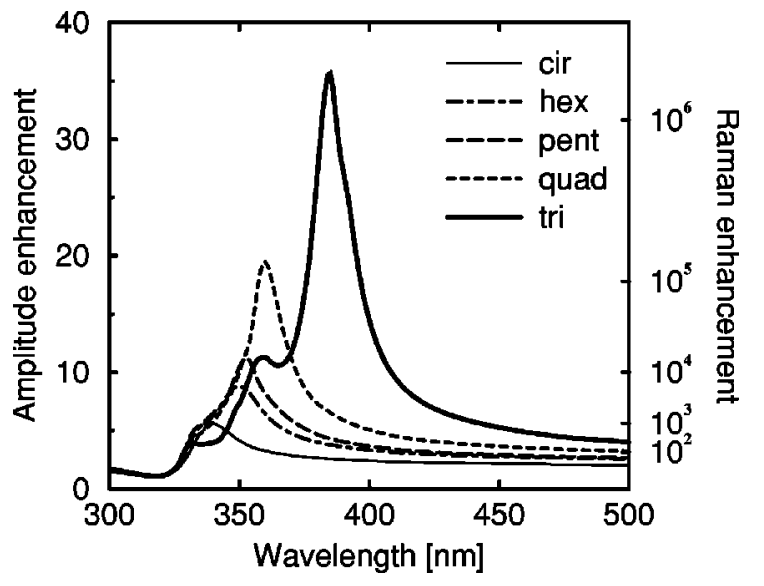

FIG. 5. Maximum amplitude enhancement $1 \mathrm{~nm}$ from the surface of the $20 \mathrm{~nm}$ simplexes. The right scale gives the Raman enhancement.

\section{Enhancement}

For molecules excited far from an electronic absorption band, the intensity of Raman scattered light is proportional to the fourth power of the local electric field amplitude where the molecule is immersed. ${ }^{3}$ Plasmon resonant particles provide a convenient method of enhancing the electromagnetic fields and therefore are ideal SERS substrates. A second enhancement mechanism, the so-called chemical enhancement, related to the adsorption of the molecule on the metal, can also contribute to SERS. ${ }^{70-72}$ However, electromagnetic enhancement is believed to play the major contribution to SERS.

The electromagnetic enhancement effectively experienced by a molecule depends on the location where the molecule is adsorbed on the metal, as well as the relative position of the Raman-active site within the molecule. This becomes very important when the molecule is large and placed in a strongly inhomogeneous field. This was recently verified experimentally by van Duyne, who inserted different numbers of nonactive linker molecules between the adsorption site of a molecule and the Raman-active site. ${ }^{73}$ In this experiment, the Raman signal was highly dependent on the number of linker molecules and, therefore, on the distance between the surface and the Raman-active site, particularly for nonregular (tetrahedral) nanoparticles. Further, when a large number of molecules are adsorbed on the same nanoparticle, a spectral shift in the particle resonances can be observed. ${ }^{74}$

In this section we will discuss the near-field distributions associated with the plasmon resonances of the simplexes, with emphasis on the local variation of the field amplitude around the particles.

Figure 5 shows the maximum enhancement obtained at a $1 \mathrm{~nm}$ distance from the surface as a function of the wavelength. The amplitude enhancement is shown on the left axis and the corresponding Raman enhancement on the right one. We observe that the field enhancement, which is a near-field quantity, is strongly correlated to the SCS, a far-field quantity (compare Figs. 1 and 5). The position of the main resonance is the same, but the resonance width is broader in the enhancement diagram (Fig. 5) than in the SCS (Fig. 1). This 


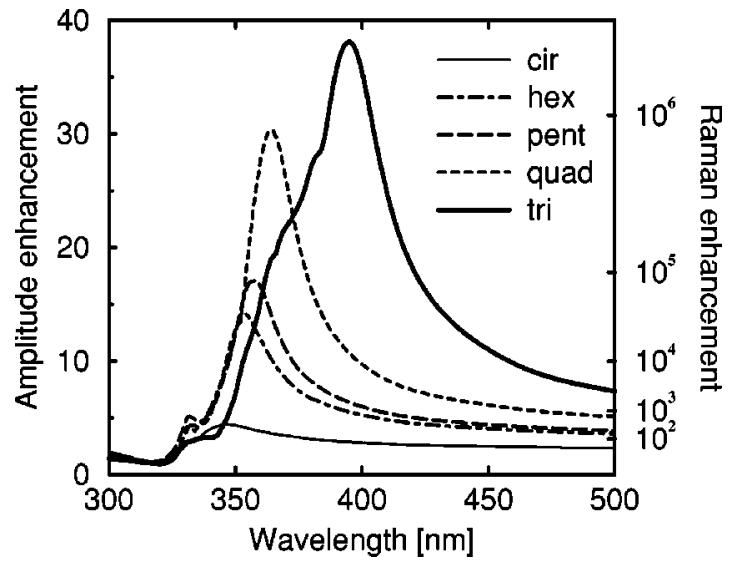

FIG. 6. Maximum amplitude enhancement $1 \mathrm{~nm}$ from the surface of the $50 \mathrm{~nm}$ simplexes. The right scale gives the Raman enhancement.

is simply because in Fig. 5 we report the maximum amplitude enhancement around the particle, whereas in Fig. 1 we report the SCS, which is related to the field amplitude squared. The maximum amplitude enhancement increases considerably for more complex shapes: Whereas it is 6 for the circle, it is about 35 for the triangle.

The differences between the simplex shapes is much more pronounced for the enhancement than for the SCS. For the triangle the maximum Raman enhancement exceeds $10^{6}$, and for the square it is still about $10^{5}$, whereas it is below $10^{4}$ for the hexagon, the pentagon, and the circle.

The field enhancement for the larger simplexes is shown in Fig. 6, again at a distance of $1 \mathrm{~nm}$ from the surface. We observe that the correlation with the SCS diagram is now weaker. The maximum enhancement is comparable to that obtained previously for the smaller particles.

As previously discussed, the local variations of the nearfield distribution determine the effective enhancement experienced by a molecule. This is investigated in Fig. 7, where we present the amplitude distribution as a function of the distance from the tip surface, for the triangle, square, and circle. The data correspond to the main resonance, and two particle sizes are considered.

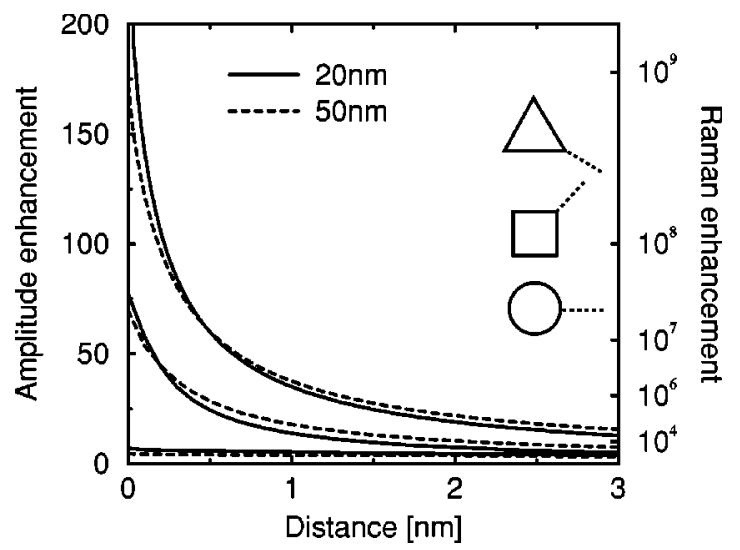

FIG. 7. Enhancement as a function of the distance from the corner (along the dashed line in the inset), for the main resonance of the $20 \mathrm{~nm}$ and $50 \mathrm{~nm}$ simplexes.
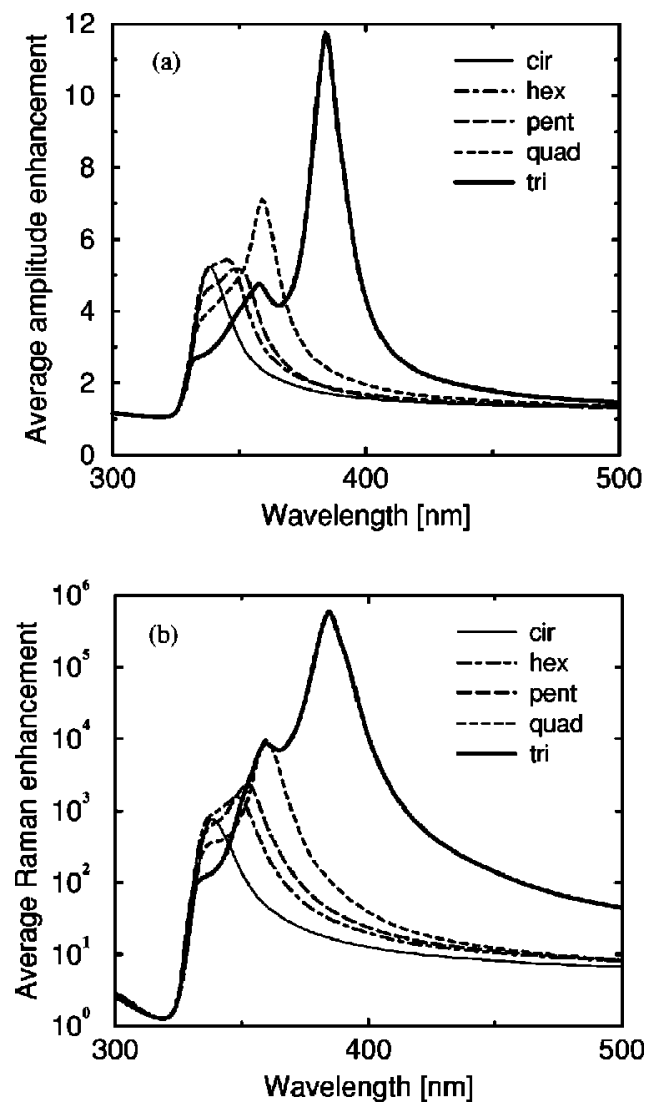

FIG. 8. Average enhancements, $1 \mathrm{~nm}$ from the surface of the $20 \mathrm{~nm}$ simplexes. (a) Average amplitude enhancement and (b) average Raman enhancement.

For the circle, the field amplitude on the surface reaches 6.9 (4.5), for the small (large) circle. At $2 \mathrm{~nm}$ from the surface, the amplitude drops to 2.2 (1.7). For the triangle we observe a huge enhancement for both particle sizes: near the surface more than 100 in terms of amplitude $\left(10^{8}\right.$ Raman) and still more than $50\left(6 \times 10^{6}\right)$ at a half-nanometer distance (Fig. 7). For the square we obtain a similar behavior, although the amplitudes are about a factor of 2 smaller (the Raman enhancement being 16 times weaker). The strongfield amplitude variations for such nonregular structures might explain the "hot spots" observed both in SERS experiments and in direct measurement of the locally enhanced field. ${ }^{5,75}$

Figure 7 indicates similar results for the maximum amplitude distribution around the $20 \mathrm{~nm}$ and the $50 \mathrm{~nm}$ simplexes. Although not shown here, this enhancement rapidly decreases for particle sizes above $50 \mathrm{~nm} .{ }^{50}$ For example, the maximum amplitude enhancement at the corner of a $100 \mathrm{~nm}$ triangle is only half that of the 20 or $50 \mathrm{~nm}$ particles.

Within our model we therefore observe that for a given particle shape the maximum field enhancement increases with decreasing particle sizes down to $50 \mathrm{~nm}$ and then remains fairly constant. Note that this maximum enhancement occurs at different wavelengths for different sizes.

Although the local maximum enhancement is similar for particles in the $20-50 \mathrm{~nm}$ range, the average over the entire particle may differ with the particle size. This is important 

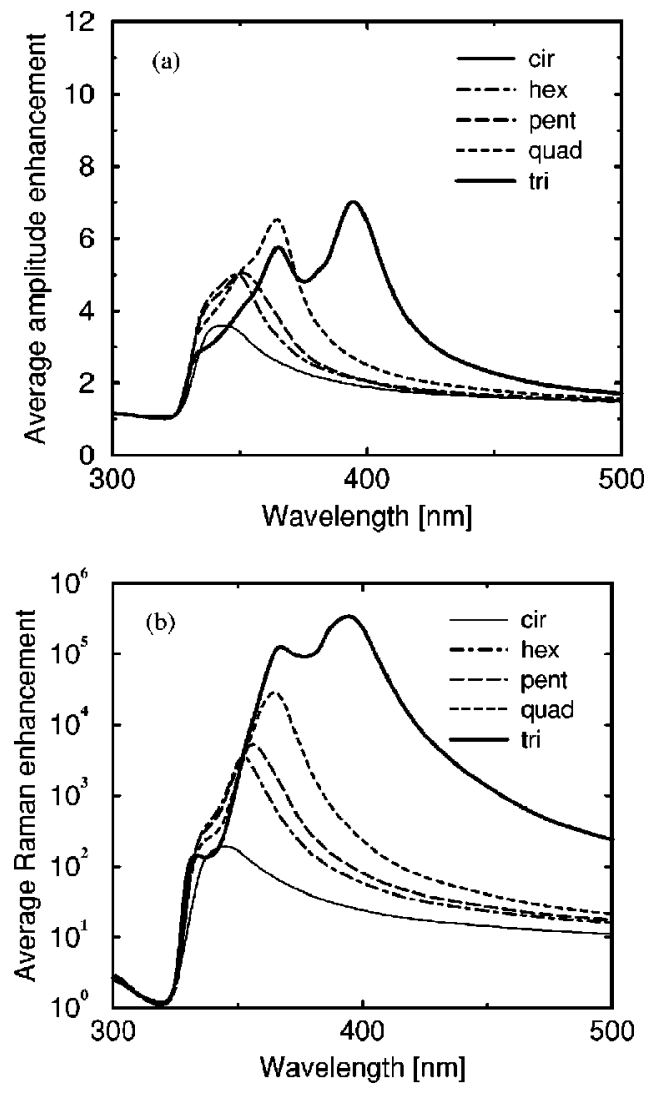

FIG. 9. Average enhancements, $1 \mathrm{~nm}$ from the surface of the $50 \mathrm{~nm}$ simplexes. (a) Average amplitude enhancement and (b) average Raman enhancement.

for SERS experiments in which large numbers of molecules are used, since the measured Raman signal is proportional to the average Raman enhancement on the surface. This is illustrated in Fig. 5 of Ref. 51, where the overall near-field distribution for particles between 10 and $100 \mathrm{~nm}$ is shown. We shall now discuss this average enhancement in further details.

In Fig. 8 we show the averaged enhancement for the $20 \mathrm{~nm}$ simplexes. This average value is obtained by taking between 500 and 800 points (depending on the particle shape) distributed regularly around the particle, at a $1 \mathrm{~nm}$ distance from the surface. Note that the average amplitude [Fig. 8(a)] and the average Raman enhancement [Fig. 8(b)] must now be represented on separate graphs.

In Fig. 8(a) we observe that the average field amplitude is strongly correlated with the SCS (Fig. 1), both with respect to the wavelength and the width of the different resonances. The average field amplitude enhancement is quite similar for the circle, the hexagon, and the pentagon: about 5.5 times the initial amplitude. For the square it is about 7.5 at the corresponding main resonance wavelength, whereas it reaches almost 12 for the triangle.

The average Raman enhancement, as shown in Fig. 8(b), is less than $10^{3}$ for the circle, becomes then larger for the hexagon and the pentagon, and reaches about $10^{4}$ for the square and almost $10^{6}$ for the triangle (always at the corresponding main resonance wavelength). It is important to re-

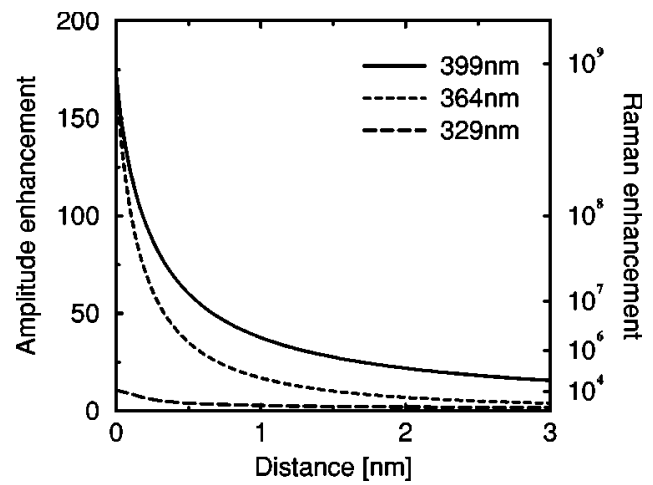

FIG. 10. Field amplitude enhancement as a function of the distance from the corner of the $50 \mathrm{~nm}$ triangle, for three different resonance wavelengths.

alize that, due to the rapid variations of the field, the maximum field amplitude on the particle circumference dominates the average Raman enhancement, as the fourth power of the field is taken. This is the reason why the average Raman enhancement is larger than the fourth power of the corresponding average field enhancement [compare Figs. 8(a) and 8(b)].

Similar results for the $50 \mathrm{~nm}$ simplexes are shown in Fig. 9. Comparing Figs. 8(a) and 9(a), we observe that these larger simplexes produce a smaller average field enhancement than their $20 \mathrm{~nm}$ counterparts. This is particularly the case for the triangular wires, where the maximum average enhancement drops from almost 12 to less than 7 times the illumination amplitude. (For the other shapes, the decrease of the maximum average enhancement is less than 15\%.) For the average Raman enhancement we also observe a smaller enhancement for the $50 \mathrm{~nm}$ circular particle than for its $20 \mathrm{~nm}$ counterpart. For the hexagon, pentagon, and square particles, the average Raman enhancement is somewhat larger for the $50 \mathrm{~nm}$ than for the $50 \mathrm{~nm}$ simplexes [Figs. 8(b) and 9(b)]. For the triangular particle, the average Raman enhancement at the main resonance is slightly weaker for the $50 \mathrm{~nm}$ particle than for the $20 \mathrm{~nm}$ one, whereas for the next resonance it is more than 10 times stronger. Again, this can be understood with the larger maximum Raman enhancement, as observed in Figs. 5 and 6: This larger maximum value outweighs the fact that, for the $50 \mathrm{~nm}$ particles, the field amplitude is in average smaller than for the $20 \mathrm{~nm}$ particles.

This illustrates the complexity of the interpretation of Raman experiments in which large numbers of molecules are used. The fact that the local Raman enhancement (Figs. 5 and 6) can be much stronger than the average enhancement [Figs. 8(b) and 9(b)] indicates that in such an experiment a very limited number of molecules can contribute the major part of the SERS effect. Let us finally note that the distance between the active Raman site and the surface can also influence the respective magnitude of the local and average enhancements. As a matter of fact, Fig. 7 indicates that at very short distances from the surface, the field is stronger for the $20 \mathrm{~nm}$ simplexes. In that case, both the average amplitude and the average Raman enhancements are larger for the $20 \mathrm{~nm}$ simplexes. 


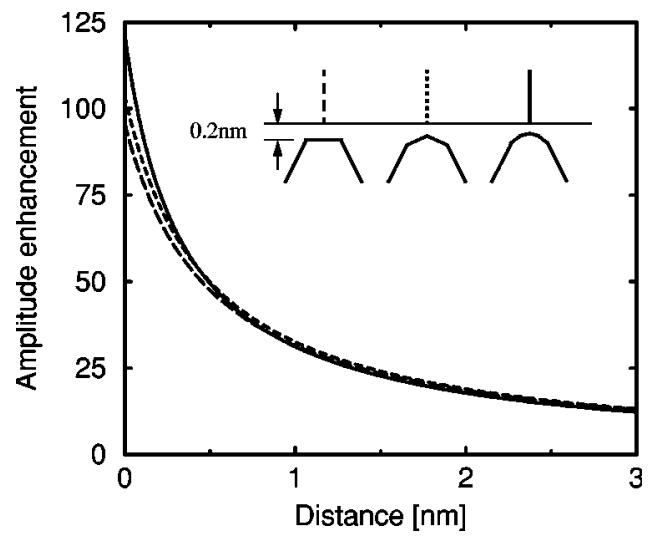

FIG. 11. Field distribution at the vicinity of the corner of the $20 \mathrm{~nm}$ triangle, at the main resonance. Three different discretization schemes for the rounded-off corner are investigated. The field is computed along the line, starting $0.2 \mathrm{~nm}$ from the rounded-off corner.

\section{Spectral response}

The resonance spectra of nonregular particles are more complex than those of regular particles. The possibility to address different resonances by tuning the illumination wavelength can prove useful for specific applications. In Fig. 10 we show the field amplitude as a function of the distance from the corner for the three resonances of the $50 \mathrm{~nm}$ triangle (see Fig. 4). We observe a similar enhancement of about 150 close to the surface for the main resonance $(\lambda$ $=399 \mathrm{~nm}$ ) and the resonance at $\lambda=364 \mathrm{~nm}$. However, the field decays more rapidly for the latter resonance (Fig. 10). This behavior can be related to the polarization charge distribution associated with both resonances, as discussed in Sec. III A and illustrated by the animations in Ref. 52. For the main resonance $(\lambda=399 \mathrm{~nm})$ a pointlike charge distribution builds at the corner. This distribution produces a field amplitude dependence in $1 / d$, where $d$ is the distance from the surface. The resonance at $\lambda=364 \mathrm{~nm}$ is associated with a dipolarlike charge distribution, leading to a $1 / d^{2}$ distance dependence. Keep in mind that we have a 2D geometry, so the pointlike and dipolar charge distributions correspond to 2D distributions. ${ }^{6}$

For the small resonance at $\lambda=329 \mathrm{~nm}$ we show the field amplitude as a function of the distance from the longitudinal corner, where the field is maximum [see Fig. 3(c)]. The amplitude maximum with about 10 is moderate and at the corner the field decays rather slowly, compared to the other resonances.

\section{Influence of the dielectric function}

As mentioned in the Introduction, a more sophisticated model of the material would include the modification of the bulk permittivity for very small particle sizes, as the electrons mean free path is reduced by scattering at the surface. Let us briefly discuss how this would affect our results.

Kreibig found that for silver particles near the plasma frequency the imaginary part $\varepsilon^{\prime}$ of the permittivity is given by $^{76}$

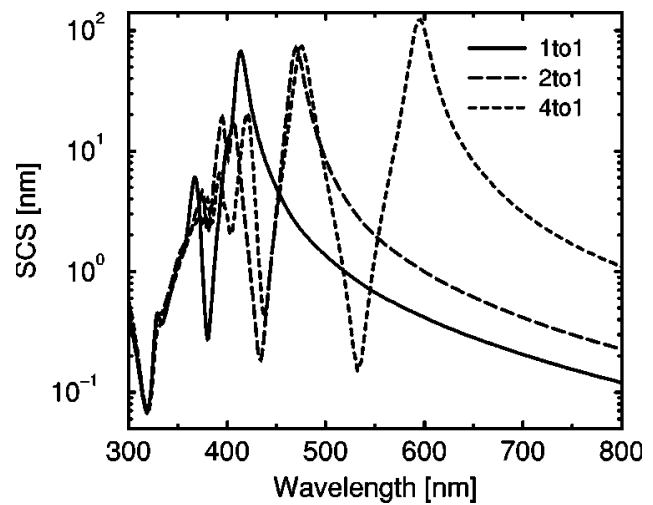

FIG. 12. SCS's for three right-angled triangles with the same area and a different perpendicular to base ratio.

$$
\varepsilon^{\prime}=0.23+2.64 / A
$$

with $A$ the particle size in nanometers. The real part of the permittivity, which determines at which wavelengths the resonances occur, is nearly unaffected. ${ }^{76}$ This means that for a $12 \mathrm{~nm}$ particle, the complex part of the permittivity is almost doubled with respect to the bulk value. Using this modified value, the resonance would be broader, and the intensity of the scattered near and far field would be approximately half that obtained with the bulk value. For the $20 \mathrm{~nm}$ particles discussed here, the result would be accordingly less affected, whereas for $50 \mathrm{~nm}$ the results would barely be affected.

Taking this size-dependent permittivity, one might ask which particle size gives the largest enhancement. We would then have a tradeoff between the decrease of the enhancement due to retardation effects for larger particles (which sets in for particles larger than $50 \mathrm{~nm}$ ) and the increase of the microscopic absorption for very small particles. Taking these two effects into account, we estimate the maximum enhancement to happen for particles in the 30-40 nm range.

\section{Singular behavior at corners}

Dealing with sharp corners introduces additional numerical difficulties since the field becomes singular at short distances from an infinitely sharp, perfectly conducting corner. ${ }^{77}$ However, the sharpness of a real particle is limited by surface and boundary energies. Therefore we have rounded off each corner by $0.25 \mathrm{~nm}$, providing a more realistic model. Nonetheless, our results indicate that plasmon resonances also produce extremely strong fields at the particle corners. It was therefore important to verify that the discretization of the corners does not introduce any numerical artifacts.

In Fig. 11 we present the field amplitude near the corner of the $20 \mathrm{~nm}$ triangle at main resonance for the three different corner discretization schemes shown in the inset. We observe that the field near the corner is only slightly affected by the discretization. The somewhat larger field obtained for the smoothest discretization (solid line, Fig. 11) can be related to the fact that the field is effectively computed at a shorter distance from the surface (see the inset in Fig. 11). Besides 


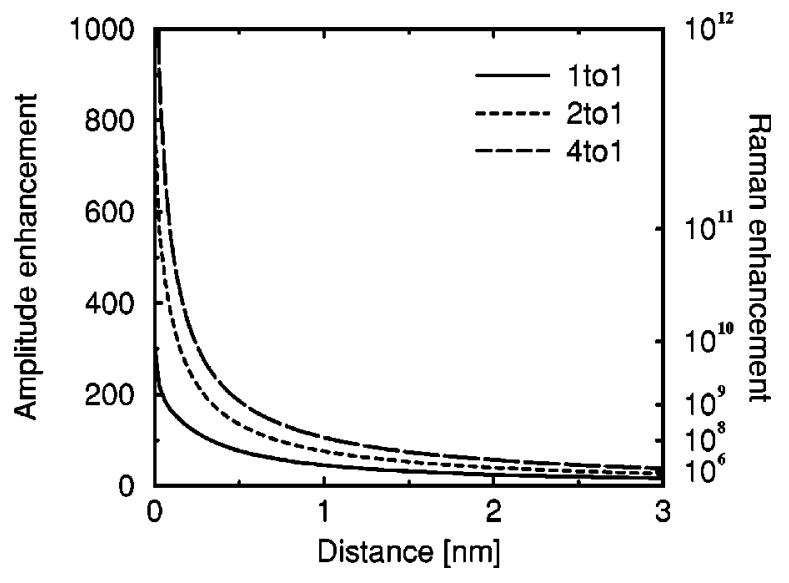

FIG. 13. Amplitude enhancement at the main resonance, near the sharp corner of right-angled triangles with the same area and a different perpendicular to base ratio.

the stability of the method this also demonstrates that the major role is played by the overall particle shape and not by such a small detail as the discretization of one corner. Note that the SCS diagram is not at all affected by the discretization scheme (not shown).

\section{E. Other geometries}

We will now break the symmetry of the triangular simplex. In Fig. 12 we present the SCS diagram for three rightangled triangles, with different perpendicular to base aspect ratios. Again, the area of the triangles is the same as that of the $20 \mathrm{~nm}$ circle. The illumination direction is normal to the hypotenuse.

In Fig. 12 we now observe a much more complex structure than for the equilateral triangle. The main resonance is strongly shifted to higher wavelengths, namely, from $\lambda$ $=385 \mathrm{~nm}$ (equilateral) to $\lambda=414 \mathrm{~nm}$ (1 to 1$), \lambda$ $=470 \mathrm{~nm}$ (2 to 1$)$, and $\lambda=596 \mathrm{~nm}$ (4 to 1$)$. The main resonance is therefore shifted into the red optical range for the 4 to 1 particle (note the different wavelength range in Fig. 12).

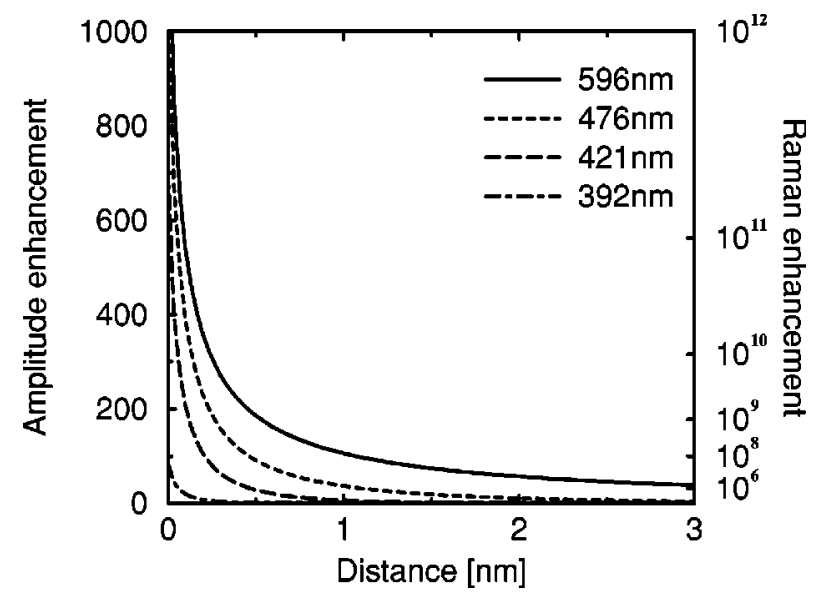

FIG. 14. Amplitude enhancement as a function of the distance from the sharp corner of the 4 to 1 triangle, for four different resonance wavelengths (see Fig. 12).

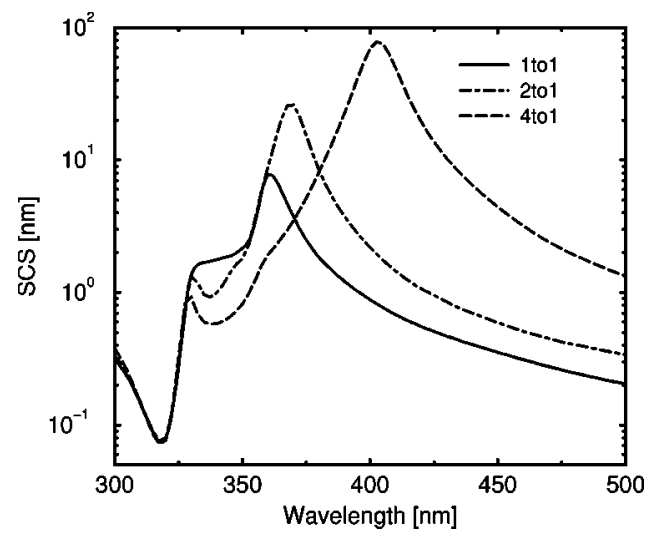

FIG. 15. SCS's for three rectangular particles with the same area and a different perpendicular to base ratio.

The SCS at the main resonance also increases slightly for higher aspect ratios. Moreover, the higher-order resonances become more numerous and more pronounced for higher aspect ratios. Note that a similar redshifted main resonance and larger SCS with increasing aspect ratio was recently observed experimentally for gold nanorods. ${ }^{78}$

Figure 13 shows that the near-field amplitude dramatically increases for higher aspect ratios. For example at the very tip of the 4 to 1 triangle, the field enhancement exceeds 1000 , corresponding to a Raman enhancement of $10^{12}$; at a distance of $1 \mathrm{~nm}$ from the tip, the field enhancement is still larger than 100 ( $>10^{8}$ Raman).

Finally, we present in Fig. 14 the dependence of the field amplitude as a function of the distance from the sharp corner of the 4 to 1 triangle for four different resonances. The three resonances at $\lambda=596 \mathrm{~nm}$ (order 0 ), $\lambda=476 \mathrm{~nm}$ (order 1), and $\lambda=421 \mathrm{~nm}$ (order 2) produce a similar enhancement on the particle surface and then decay differently. This is similar to the behavior observed for the order- 0 and - 1 resonances of the triangular simplex (Fig. 10). As expected, the highest order mode $(\lambda=421 \mathrm{~nm})$ has the fastest decay, as it is associated with a triple polarization charge distribution (e.g., $+\quad+\quad)$ near the corner. The amplitude of the resonance at

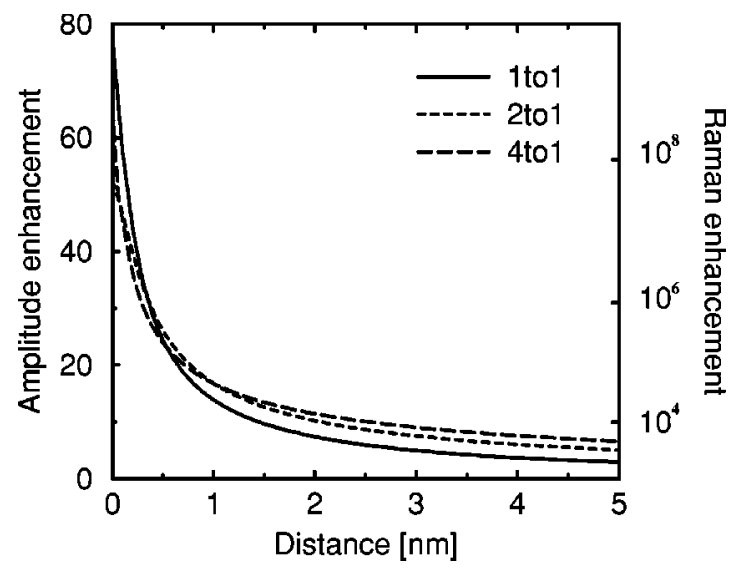

FIG. 16. Amplitude enhancement at the main resonance, near the transverse corner of rectangular particles with the same area and a different perpendicular to base ratio (SCS's in Fig. 15). 
$392 \mathrm{~nm}$ is rather moderate. For this resonance, polarization charges of different sign constitute also near the lower-right corner.

Although the aspect ratio influences the spectral response of the particle, its cross-sectional shape remains the dominant factor, as illustrated in Fig. 15, where we show the SCS's for rectangular particles with increasing aspect ratio. These particles have the same area as the triangles investigated in Fig. 12, corresponding to the $20 \mathrm{~nm}$ simplexes (Fig. 1).

Again, the main resonance is redshifted for increasing aspect ratio, although this shift amounts less than $100 \mathrm{~nm}$ between a square and a 4 to 1 elongated particle (Fig. 15). This is much less than for the right-angled particles in Fig. 12. On the other hand, the SCS at the main resonance strongly increases with increasing aspect ratio, reaching similar values as the right-angled particles (compare Figs. 15 and 12).

In Fig. 16 we show the field enhancement associated with different aspect ratio rectangular particles. This enhancement is a factor of 10 weaker than that of triangular particles (compare Fig. 16 with Fig. 13). Quite interestingly, at very short distances from the particle, the field is strongest for the square particle (1 to 1 , Fig. 16). This is probably related to the strongest charges confinement that can occur on both sides of the corner for a square particle. At larger distances from the particle the field enhancement is larger for the higher aspect ratio particles (Fig. 16). This indicates that, although not as much confined, the amount of induced polarization charges is larger, in agreement with the larger SCS.

\section{CONCLUSION}

Our results reveal the complexity of the plasmon resonances in 2D nanoparticles (nanowires) with a nonregular cross section. We have shown that the resonance spectrum strongly depends on the particle shape. The higher the particle symmetry, the simplest its spectrum (e.g., a small cylindrical particle exhibits only one resonance, whereas a square has two and an equilateral triangle at least three distinct resonances). Several additional resonances are observed for right-angled triangular particles with a high perpendicular to base ratio.

These complex scattering cross sections are also associated with a dramatic near-field enhancement at close vicinity of the particle. We found the strongest enhancement for particles with dimensions smaller than $50 \mathrm{~nm}$. Right-angled triangular particle produce a field amplitude exceeding 1000 times that of the incident field at short distances from the surface. This dramatic near-field enhancement corresponds to a Raman enhancement in the order of $10^{12}$. Such enhancement can help understand recent SERS experiments, where single-molecule detection was possible, thereby requiring an enhancement factor similar to that we computed. ${ }^{4-6}$ The strong localization of the near field at specific positions around the metallic particle, as well as its rapid decay when one moves further away from the particle surface, can also explain the "hot spots" observed in SERS experiments, where specific sites appear to be particularly active. This is also confirmed by our results on the average Raman enhancement, which indicate that a limited number of molecules can contribute the major part of the SERS signal, even in experiments in which large numbers of molecules are used.

The spectral response of these nonregular particles could also be used for near-field optical microscopy,${ }^{69}$ nonradiative optical transfer, ${ }^{13,14}$ and for building new active optical components. ${ }^{16}$

Although we focused the present article on individual, noninteracting particles with a nonregular shape, it should be emphasized that similar Raman enhancements can be observed in interacting, regularly shaped particles, as recently investigated in Refs. 79 and 80.

This work should help design nanoparticles with tailored plasmon resonances for specific applications.

\section{ACKNOWLEDGMENTS}

This work was supported by the Swiss National Science Foundation and by the U.S. NSF (NSF-DMR-96-23949 and NSF-DMR-97-24535).
*Correspondence author. Electronic address: martin@ifh.ee.ethz.ch

${ }^{1}$ M. Kerker, Appl. Opt. 30, 4699 (1991).

${ }^{2}$ H. Metiu, Prog. Surf. Sci. 17, 153 (1984).

${ }^{3}$ M. Moskovits, Rev. Mod. Phys. 57, 783 (1985).

${ }^{4}$ K. Kneipp, Y. Wang, H. Kneipp, L.T. Perelman, I. Itzkan, R.R. Dasari, and M.S. Feld, Phys. Rev. Lett. 78, 1667 (1997).

${ }^{5}$ S. Nie and S.R. Emory, Science 275, 1102 (1997).

${ }^{6}$ H. Xu, J. Aizpurua, M. Käll, and P. Apell, Phys. Rev. E 62, 4318 (2000).

${ }^{7}$ R. Elghanian, J.J. Storhoff, R.C. Mucic, R.L. Letsinger, and C.A. Mirkin, Science 277, 1078 (1997).

${ }^{8}$ L.A. Lyon, M.D. Musick, and M.J. Natan, Anal. Chem. 70, 5177 (1998)

${ }^{9}$ S. Schultz, D.R. Smith, J.J. Mock, and D.A. Schultz, Proc. Natl. Acad. Sci. U.S.A. 97, 996 (2000).

${ }^{10}$ J.C. Hulteen, D.A. Treichel, M.T. Smith, M.L. Duval, T.R.
Jensen, and R.P. van Duyne, J. Phys. Chem. B 103, 3854 (1999).

${ }^{11}$ T.J. Silva and S. Schultz, Rev. Sci. Instrum. 67, 715 (1996).

${ }^{12}$ R.M. Stöckle, Y.D. Suh, V. Deckert, and R. Zenobi, Chem. Phys. Lett. 318, 131 (2000).

${ }^{13}$ J.R. Krenn, A. Dereux, J.C. Weeber, E. Bourillot, Y. Lacroute, J.P. Goudonnet, G. Schider, W. Gotschy, A. Leitner, F.R. Aussenegg, and C. Girard, Phys. Rev. Lett. 82, 2590 (1999).

${ }^{14}$ J.-C. Weeber, A. Dereux, C. Girard, J.R. Krenn, and J.-P. Goudonnet, Phys. Rev. B 60, 9061 (1999).

${ }^{15}$ J.R. Krenn, J.C. Weeber, E. Bourillot, A. Dereux, J.P. Goudonnet, G. Schider, A. Leitner, F.R. Aussenegg, and C. Girard, Phys. Rev. B 60, 5029 (1999).

${ }^{16}$ J. Tominaga, C. Mihalcea, D. Büchel, H. Fukuda, T. Nakano, N. Atoda, H. Fuji, and T. Kikukawa, Appl. Phys. Lett. 78, 2417 (2001).

${ }^{17}$ K. Bromann, C. Félix, H. Brune, W. Harbich, R. Monot, J. Buttet, 
and K. Kern, Science 274, 956 (1996).

${ }^{18}$ D.M. Kolb, R. Ullmann, and T. Will, Science 275, 1097 (1997).

${ }^{19}$ Y. Yu, S. Chang, C. Lee, and C.R.C. Wang, J. Phys. Chem. B 101, 6661 (1997).

${ }^{20}$ K. Abe, T. Hanada, Y. Yoshida, N. Tanigaki, H. Takiguchi, H. Nagasawa, M. Nakamoto, T. Yamaguchi, and K. Yase, Thin Solid Films 327-329, 524 (1997).

${ }^{21}$ D.Y. Petrovykh, F.J. Himpsel, and T. Jung, Surf. Sci. 407, 189 (1998).

${ }^{22}$ G.L. Che, B.B. Lakshmi, E.R. Fisher, and C.R. Martin, Nature (London) 393, 346 (1998).

${ }^{23}$ J. Viereck, F. Stietz, M. Stuke, T. Wenzel, and F. Träger, Surf. Sci. 383, 749 (1999).

${ }^{24}$ J. Bosbach, Appl. Phys. Lett. 74, 2605 (1999).

${ }^{25}$ I. Utke, P. Hoffmann, B. Dwir, E. Kapon, and P. Doppelt, J. Vac. Sci. Technol. B 18, 3168 (2000).

${ }^{26}$ A.P. Li, F. Müller, and U. Gösele, Electrochem. Solid-State Lett. 3, 131 (2000).

${ }^{27}$ D.-S. Wang, H. Chew, and M. Kerker, Appl. Opt. 19, 2256 (1980)

${ }^{28}$ M. Kerker, D.-S. Wang, and H. Chew, Appl. Opt. 19, 4159 (1980).

${ }^{29}$ P.K. Aravind, A. Nitzan, and H. Metiu, Surf. Sci. 110, 189 (1981).

${ }^{30}$ P.W. Barber, R.K. Chang, and H. Massoudi, Phys. Rev. B 27, 7251 (1983).

${ }^{31}$ M. Inoue and K. Ohtaka, J. Phys. Soc. Jpn. 52, 1457 (1983).

${ }^{32}$ K.T. Carron, W. Fluhr, M. Meier, A. Wokaun, and H.W. Lehmann, J. Opt. Soc. Am. B 3, 430 (1986).

${ }^{33}$ R. Rojas and F. Claro, J. Chem. Phys. 98, 998 (1993).

${ }^{34}$ A.I. Vanin, J. Appl. Spectrosc. 62, 32 (1995).

${ }^{35}$ K.-P. Charlé, L. König, S. Nepijko, I. Rabin, and W. Schulze, Cryst. Res. Technol. 33, 1085 (1998).

${ }^{36}$ J. Gersten and A. Nitzan, J. Chem. Phys. 73, 3023 (1980).

${ }^{37}$ M. Weber and D.L. Mills, Phys. Rev. B 27, 2698 (1983).

${ }^{38}$ N. García, G. Díaz, J.J. Saénz, and C. Ocal, Surf. Sci. 143, 342 (1984)

${ }^{39}$ F.J. García-Vidal and J.B. Pendry, Prog. Surf. Sci. 50, 55 (1995).

${ }^{40}$ S.J. Oldenburg, R.D. Averitt, S.L. Westcott, and N.J. Halas, Chem. Phys. Lett. 288, 243 (1998).

${ }^{41}$ F.J. García-Vidal and J.B. Pendry, Phys. Rev. Lett. 77, 1163 (1996)

${ }^{42}$ L. Salomon, F. de Fornel, and P.M. Adam, J. Opt. Soc. Am. B 16, 2695 (1999).

${ }^{43}$ M. Kahl, E. Voges, S. Kostrewa, C. Viets, and W. Hill, Sens. Actuators B 51, 285 (1998).

${ }^{44}$ M. Kahl and E. Voges, Phys. Rev. B 61, 14078 (2000).

${ }^{45}$ R. Fuchs, Phys. Rev. B 11, 1732 (1975).

${ }^{46}$ W.-H. Yang, G.C. Schatz, and R.P. van Duyne, J. Chem. Phys. 103, 869 (1995).

${ }^{47}$ T.R. Jensen, G.C. Schatz, and R.P. van Duyne, J. Phys. Chem. B 103, 2394 (1999).

${ }^{48}$ N. Félidj, J. Aubard, and G. Lévi, J. Chem. Phys. 111, 1195 (1999).
${ }^{49}$ J.P. Kottmann and O.J.F. Martin, IEEE Trans. Antennas Propag. 48, 1719 (2000).

${ }^{50}$ J.P. Kottmann, O.J.F. Martin, D.R. Smith, and S. Schultz, Chem. Phys. Lett. 341, 1 (2001).

${ }^{51}$ J.P. Kottmann, O.J.F. Martin, D.R. Smith, and S. Schultz, Opt. Express 6, 213 (2000).

${ }^{52}$ J.P. Kottmann, O.J.F. Martin, D.R. Smith, and S. Schultz, New J. Phys. 2, 27.1 (2000).

${ }^{53}$ C.F. Bohren and D. R. Huffman, Absorption and Scattering of Light by Small Particles (Wiley, New York, 1983).

${ }^{54}$ U. Kreibig and M. Vollmer, Optical Properties of Metal Clusters, Springer Series in Material Science Vol. 25 (Springer Verlag, Berlin, 1995).

${ }^{55}$ R. Ruppin, in Electromagnetic Surface Modes, edited by A.D. Boardman (Wiley, Chichester, 1982), Chap. 9.

${ }^{56}$ F. Sauter, Z. Phys. 203, 488 (1967).

${ }^{57}$ F. Forstmann, Z. Phys. B 32, 385 (1979).

${ }^{58}$ U. Kreibig and C.v. Fragstein, Z. Phys. 224, 307 (1969).

${ }^{59}$ L. Genzel, T.P. Martin, and U. Kreibig, Z. Phys. B 21, 339 (1975).

${ }^{60}$ J.-Y. Bigot, V. Halté, J.C. Merle, and A. Daunois, Chem. Phys. 251, 181 (2000).

${ }^{61}$ M. Brack, Rev. Mod. Phys. 65, 677 (1993).

${ }^{62}$ V. Kresin, Phys. Rep. 220, 1 (1992).

${ }^{63}$ V. Bonacic-Koutecky, P. Fantucci, and J. Koutecky, Chem. Rev. 91, 1035 (1991).

${ }^{64}$ P.B. Johnson and R.W. Christy, Phys. Rev. B 6, 4370 (1972).

${ }^{65}$ O.J.F. Martin and N.B. Piller, Phys. Rev. E 58, 3909 (1998).

${ }^{66}$ M. Paulus, P. Gay-Balmaz, and O.J.F. Martin, Phys. Rev. E 62, 5797 (2000).

${ }^{67}$ M. Paulus and O.J.F. Martin, J. Opt. Soc. Am. A 18, 854 (2001).

${ }^{68}$ J.D. Jackson, Classical Electrodynamics, 3rd ed. (Wiley, New York, 1999).

${ }^{69}$ J.P. Kottmann, O.J.F. Martin, D.R. Smith, and S. Schultz, J. Microsc. 202, 60 (2001).

${ }^{70}$ A. Otto, I. Mrozek, H. Grabhorn, and W. Akemann, J. Phys. C 4, 1143 (1992).

${ }^{71}$ P. Kambhampati, C.M. Child, M.C. Foster, and A. Campion, J. Chem. Phys. 108, 5013 (1998).

${ }^{72}$ A. Campion and P. Kambhampati, Chem. Soc. Rev. 27, 241 (1998).

${ }^{73}$ R.P. van Duyne (private communication).

${ }^{74}$ M.D. Malinsky, L. Kelly, G.C. Schatz, and R.P. van Duyne, J. Am. Chem. Soc. 123, 1471 (2001).

${ }^{75}$ P. Gadenne, X. Quelin, S. Ducourtieux, S. Gresillon, L. Aigouy, J.-C. Rivoal, V. Shalaev, and A. Sarychev, Physica B 279, 52 (2000).

${ }^{76}$ U. Kreibig and C. von Fragstein, J. Phys. F 4, 999 (1974).

${ }^{77}$ J. van Bladel, Singular Electromagnetic Fields and Sources (Clarendon, Oxford, 1991).

${ }^{78}$ M.B. Mohamed, V. Volkov, S. Link, and M.A. El-Sayed, Chem. Phys. Lett. 317, 517 (2000).

${ }^{79}$ J.P. Kottmann and O.J.F. Martin, Opt. Express 8, 655 (2001).

${ }^{80}$ J.P. Kottmann and O.J.F. Martin, Opt. Lett. 26, 1096 (2001). 